International Journal on Cloud Computing: Services and Architecture (IJCCSA) ,Vol.3, No.3, June 2013

\title{
A FRAMEWORK FOR ERP SYSTEMS IN SME BASED on Cloud Computing TeCHNOlogy
}

\author{
Ahmed A. Al-Johani ${ }^{1}$ and Ahmed E. Youssef ${ }^{2}$ \\ ${ }^{1}$ CCIS, King Saud University, Riyadh, KSA \\ al johaniaa@yahoo.com \\ ${ }^{2}$ CCIS, King Saud University, Riyadh, KSA \\ Faculty of Engineering, Helwan University, Cairo, Egypt \\ ahyoussef@ksu.edu.sa
}

\begin{abstract}
Present Enterprise Resource Planning (ERP) offers expensive models which are hard to implement in Small and Medium Enterprises (SME) due to budget limitations. Moreover, current ERP systems are associated with several issues such as mutual synchronization of multi-typed resources, limited customization, bulky upgrading cost, solution integration, industry functionality, backup hedge and technology updates. These issues render ERP systems implementation painful, complex and time consuming and generate the necessity of a massive change in ERP framework to enhance ERP systems infrastructure and functionality. Cloud Computing (CC) platforms possess the ability to overcome ERP systems discrepancies with cost-effective, customized and highly available computing resources. The goal of this research is to merge ERP and CC benefits together to reduce the factor of expenditure cost and implementation delays through a proposed framework. For this purpose, we firstly analyze the prominent issues in current ERP systems through a comprehensive comparison between ERP before and after moving to CC environment. Secondly, we propose a generic framework for "Cloud-based ERP systems" in favour of SME. The proposed framework follows multi-instance based cloud infrastructure that initiates different ERP instances for different industries. Finally, the feasibility and efficiency of the proposed framework is tested through the study of the ERP system in "Awal" Company.
\end{abstract}

\section{KEYWORDS}

Cloud Computing (CC), Enterprise Resource Planning (ERP), Solution Integration, Industry Functionality, Small and Medium Enterprises (SME), Service-Oriented Architecture (SOA).

\section{INTRODUCTION}

Enterprise Resource Planning (ERP) systems are the ultimate aspiration of the business community as they provide collaboration with partners, external applications and information systems. However, ERP solutions have many issues and challenges such as massive upgrading cost, integration among different components, fair utilization of resources, visibility, management change, financial performance tracking, bidding and quoting etc. Due to these problems, a radical change is required within ERP framework to enhance ERP infrastructural and functionality.

Recent trends in ERP invoke that business functions and components should be service-enabled. Since ERP applications are heterogeneous in nature, they are hard to monitor in execution phase. The employment of Service Oriented Architecture (SOA) is able to escape this discrepancy as it provides smooth continuous integration between applications, protocols and platforms [1, 27, 2, $5,7]$. Moreover, the implementation of ERP through SOA approach provides third party support in management activities, planning, and provision of application services through virtual suppliers. 
SOA can be best implemented in a Cloud Computing (CC) environments. CC $[15,16]$ is the best suited option to overcome ERP systems discrepancies. Clouds can be classified into public, private, community and hybrid clouds. In public clouds, the infrastructure and other cloud services are made available to the general public over the Internet. The cloud is owned and managed by a Cloud Service Provider (CSP) who offers services to consumers on a pay-per-use basis [28]. Private cloud's infrastructure is owned and used by a single organization. It can be managed by the organization itself or by a third party internally or externally. In community cloud, infrastructure is shared between many organizations with common concerns such as security, policy, mission, and compliance. These clouds are managed and hosted internally or externally through a third party. Hybrid cloud is a combination of two or more cloud infrastructures that remain unique entities, but are bounded together to provide advantages of multiple cloud structure [3].

$\mathrm{CC}$ offers several services such as Infrastructure-as-a-Services (IaaS), Platform-as-a-Service (PaaS) and Software-as-a-Service (SaaS). SaaS provides complete applications on CC environment. With PaaS, customers can develop, test, host, deploy and maintain their applications in the same integrated environment. With IaaS, customers use cloud infrastructure according to their demands for particular time and pay only for what they use. CC environments offer many interesting characteristics that make them promising for future IT applications and services. Among these characteristics are: On-demand self-service, broad network access, resource pooling, rapid elasticity, measured services, multitenancy, scalability, economies of scale, cost effectiveness, customization, efficient resource utilization, maintainability, collaboration, virtualization, green technology, and high performance [28].

Cloud-based ERP systems facilitate extra ordinary benefits with valuable cost reduction by reducing the need for IT support team, technical IT labors, requirements gathering and project management efforts. The core motivation of this work is to gain the cost effectiveness of cloud architecture with optimal implementations of industry functionality and solution integration challenges. Many SME are willing to increase their computing resources, but they lack this advancement due to expensive solutions. $\mathrm{CC}$ with its on demand, pay-per-use infrastructure facilitates promising cost effective solutions to these SME. Enterprises always look forward to scale up and upgrade their masses, therefore, $\mathrm{CC}$ with its scalable, high performance resource pooling offers them a blessing approach to smoothly scale up and upgrade.

This research aims at proposing an ERP framework based on SaaS concepts to multitude different applications. The charm of an ERP is to interoperate applications from different domains; therefore, the same is needed to be implemented using a SaaS concept. The most prominent motive of this research is to minimize the cost of infrastructure and hardware resources through a framework that tackles industry functionality and solution integration challenges. The proposed framework is especially designed to overcome current ERP issues through the employment of ERP within the CC environment. The solution against industry functionality and solution integration with affordable cost requires the public cloud to have different ERP instances for each industry. Any SME can confidently implement our proposed framework to overcome these issues. While the utilization of the proposed framework is a good deal for cost reduction, some $\mathrm{CC}$ issues that cannot be neglected such as security have to be addressed before the initiation and implementation of our proposed cloud-based ERP framework.

\section{BACKGROUND}

Since the beginning of software manufacture in 1960s and 1970s, many organizations used simple inventory systems that were known as Material Requirement Planning (MRP). This 
allowed managers to make estimate about raw material and production. For many productions, estimate was not possible without computer as it required various input. To handle basic functions of MRP, mainframe computers were required. To make orders and purchase between companies, Electronic Data Interchange (EDI) were used to save time and cost of paper purchase and invoices. In late 1990s, because of Y2K problem, many companies move to Enterprise Resource Planning (ERP) systems [6]. Through ERP systems, organizations can control their cost and plan in efficient way. An ERP system consists of central database, process model and a common control system. Within ERP systems multiple tasks can be performed in parallel such as finance, human resources and supply chain management. All are integrated within one system and can access data from any part. With ERP systems performance of organization and response to customer can be improved and integration and accessibility of data across database can be provided [2].

ERP systems provide many advantages regardless of their extent. Especially, an important approach of ERP is to combine business management and IT infrastructure [8]. In business enterprise model, ERP systems are in the center of the enterprise, which provides collaboration with partners, external applications and information. ERP systems reduce costs, exchange information in a better way, and reduce response time between customers and suppliers [9]. An ERP system can be operated within the enterprise with one standard application that provides integration between all supporting departments such as sales, accounting, manufacturing, human resources and supply chain [10].

Implementation in $\mathrm{CC}$ environment is a promising direction to make ERP more flexible and integrated within enterprises $[17,18]$. Through CC, enterprises with the same interest can share single ERP as per their requirements and can be mentioned under the supervision of a joint team, this reduces cost and hardware for enterprises. In addition, integration of ERP systems with SOA is a good solution for enterprises. SOA consists of a set of loosely coupled distributed services within software system operated under interlinked standard protocols and interfaces [4]. SOA provides business guidelines for creating, reusing and organizing computing components in an architectural pattern. On the other side, $\mathrm{CC}$ provides a flexible Internet platform to build their SOA solutions [6]. Together, CC and SOA provide service oriented environment where consumers use different types of services through the Internet.

\section{RELATED WORK}

Many valuable works have been conducted on ERP since last decade. In [10] Elragal et. al represented a case study that showed relationship between ERP and business performance. They identified that adaptation of ERP provides many financial and operational benefits. Another valuable work on ERP system has been done in [8].The authors studied the companies that adopt ERP systems and they focus on improvements in information flow, cost reduction, response time reduction and linkage between customers and suppliers. They indicated that organizations adopted ERP obtained more advantages than those that chose not to adopt ERP. They mentioned many benefits, but cost reduction was the most important one. They mentioned that $40 \%$ of organizations adopted ERP reduced production cost and increased productivity level.

An interesting case study on implementation of ERP was done in Indian SME by Kale et. al [11]. Based on interviews with ERP vendors, issues to SME were identified and presented. Issues identified were: perception, awareness, implementation approach, change management, cost and limited resources. They also identified some factors that should be considered before planning of system implementation such as, infrastructure resource planning, human resource planning, proper education about ERP, support from top management and proper training. Another case study related to ERP and business processes were represented by Tsai et. al [20]. They examined 
performance of ERP and process problems by using the questionnaire survey and ANOVA analysis. They indicated that to implement ERP in companies, business process should be changed. Business process and system are related to each other and their consistency should be considered with high importance. As a result, they showed that if companies have no gap between system and business process, they can achieve better ERP system performance.

Somers et al [13] studied responses from organizations that implemented ERP on the basis of Critical Success Factors (CSFs) such as, IT implementation, IT failure, management support and involvement, user training, and management change etc. With the consideration of these CSFs, organizations can effectively implement ERPs and can boost their business. Yao et al [14] proposed SOA-based integration solution for ERP and Enterprise Application Integration (EAI) to solve integration issues for companies. They presented a reference model for adaptation of existing theories. Through adaptation of SOA, system integration issues can be solved smoothly and incrementally. They analyzed the classical transaction model and concluded that it is good for single machine transaction and short-lived distributed transaction for trusted domains. This model provides Atomicity, Consistency, Isolation, and Durability (ACID) strictly.

Another valuable work has been done in [19] on integration of sensor cloud and ERP. The authors designed sensor network and ERP for integration in cloud environment. They explained how to minimize resources used by sensors and sensor reconfiguration by using ERP systems information. In their design, they used distributed decision making applications and ERP to generate information related to transmission frequency of sensors. For accessing sensors data from ERP database, they used caching and indexing methods. Through their proposed design, it is possible to manage resources and save energy using the cloud approach and ERP information.

Yaseen [12] presented an explanatory case study on factors affecting ERP implementation. He considered pharmaceutical industries that adopted ERP systems. He examined factors for successful and unsuccessful implementation of ERP in terms of organizational performance and capabilities and functions of ERP. His findings showed that capabilities of the ERP systems were not utilized properly or never utilized by pharmaceutical industries under consideration. Findings also showed that functions available in the systems were not properly used and only $10 \%$ of functions were utilized. Another reason for ERP systems' failure is that ERP implementation is costly and complex. Other factors that can also affect ERP systems either directly or indirectly are user acceptance, trust work habits, cultural attitude, and management behavior.

Isse [21] discussed adaptation of $\mathrm{CC}$ and integration for new business model. He specifically observed current business models such as SAP and their relation to CC. He identified two possible models for adaptation of $\mathrm{CC}$ in business models. In the first model, $\mathrm{CC}$ works as a new extension without disturbing running business models and in the second model, CC works parallel as continuous strategy. With the benefits of $\mathrm{CC}$, industry takes the advantage of technology to reduce costs of development. With adaptation of technologies and CC, business organizations make benefits even though risks and issues are still there. Saleem [22] discussed the benefits and drawbacks of $\mathrm{CC}$ adaptation for enterprises in term of cost and security. $\mathrm{He}$ conducted case studies with $\mathrm{CC}$ users and providers. He collected data through interviews and semi-structured interviews. Finally, data were analyzed to make a decision. His final report showed that cost and security are major concerns for enterprises, especially, the large ones.

In the light of the above review, we conclude that current ERP solutions suffer from several significant limitations as summarized in Table 1. 
Table 1: Current ERP Issues

\begin{tabular}{|l|l|}
\hline ERP Issues & Why? \\
\hline Implementation & $\begin{array}{l}\text { ERP solutions are sufficiently complex and require painful } \\
\text { implementation. The approximate ERP adoption duration with } \\
\text { default settings may take months and bulky modification may take } \\
\text { (2-5) years [23]. }\end{array}$ \\
\hline Cost & $\begin{array}{l}\text { ERP solutions are too expensive as its cost for medium size } \\
\text { organization is approximately 300 million US Dollars [24]. } \\
\text { Moreover, there are other hidden expenditure such as hiring and } \\
\text { training of new staff [23]. ERP solutions are mostly affordable by } \\
\text { large organizations only [23]. }\end{array}$ \\
\hline $\begin{array}{l}\text { Functionality and } \\
\text { customization }\end{array}$ & $\begin{array}{l}\text { One ERP may not have some specific industry functionality for } \\
\text { similar or dissimilar type of industries. Therefore, many modules or } \\
\text { requirements may be misfit with customer's organization which } \\
\text { results in waste of customization cost and time to remove the } \\
\text { deficiency of functional interfaces, input data conditions, and } \\
\text { functional reports [23]. }\end{array}$ \\
\hline Integration & $\begin{array}{l}\text { Integration between ERP and Non-ERP systems is another } \\
\text { noteworthy issue [23]. Customization offers only limited } \\
\text { modifications, therefore; new dynamic business logic for application } \\
\text { integration is needed due to tight integration among applications, } \\
\text { module and data [26]. }\end{array}$ \\
\hline
\end{tabular}

\section{OUR OBJECTIVES AND METHODOLOGY}

Complex and expensive implementation, solutions integration, industry functionality, customization cost, backup hedge and technology updates are the prominent issues with ERP implementation as discussed in Table 1. ERP offers a limited customization, therefore, large modifications are not feasible due to cost and time issues. This situation demanded the need to dynamic business logic to handle integration among applications, module and data. Industry functionality or functional requirements mostly varies from one organization to another, therefore, several required functionalities need customization that results in expensive and time consuming implementations. Due to these issues, ERP packages are considered too complex and their implementation becomes painful. In contrast to ERP systems, the cloud technology offers several benefits such as no daily backups, cost reduction, no hedge of technology updates and high processing power. However, cloud solutions for ERP are limited to public clouds and are in early development stages yet as compared to established ERP suites that have been evolving from last decade. Medium and small size organizations always have budget limitations; therefore, traditional ERP option is not feasible for them. In this situation moving ERP to the cloud environment is a good deal with cost reduction, optimal industry functionality, and effective solution integration.

This research discusses all positive and feeble characteristics associated with Cloud-based ERP systems through a comprehensive comparison of ERP before and after migration to cloud computing environment. The purpose of this comparison is to show how the idea of ERP is significant whiling running ERP on cloud platform as compared to an individual ERP package. Another objective is to propose a generic framework for Cloud-based ERP in favor of SME community. The third objective of this work is to test the feasibility and efficiency of the 
International Journal on Cloud Computing: Services and Architecture (IJCCSA),Vol.3, No.3, June 2013

proposed framework through a case study of Awal IT Service Company in order to overcome prominent issues such as industry functionality and solution integration with affordable cost.

To fulfill the first objective, previous work in ERP is analyzed and issues are identified. Cloudbased ERP is investigated in the light of cloud characteristics. A comparison between the two technologies is made in tabular format to illustrate the benefits and the cost of Cloud-based ERP and to decide when migration is beneficent. To achieve the second goal, an elegant framework is proposed in order to handle industry functionality and solution integration challenges. Multiinstance-based cloud infrastructure is suggested to initiate different ERP instances for different industries. Common applications such as accounts, CRM modules, MIS systems, Invoice, HRM systems, CMS etc. will be operated under SaaS to deliver their models. Almost all enterprise application companies will add SaaS as their major stratagem. Finally, for the third objective, we study the current discrepancies, challenges and future needs of "Awal IT Services" Company. More than one visit has been conducted to observe their working environment through unplanned interview sessions with the relevant company employees. The company infrastructure, services, remote connectivity, budget, client's feedbacks are studied from the company documentations to predict current issues and challenges associate with the company. Our framework is examined against these challenges.

\section{A COMPARATIVE ANALYSIS}

The first objective of this research is to conduct a comprehensive comparison between ERP systems before and after moving on to Cloud. For this purpose, a variety of technical and research studies are reviewed to point out the prominent issues that can be solved by moving ERP onto CC environment. Our goal is to conclude how much the idea of moving ERP onto Cloud is significant? What are the benefits of shifting ERP onto Cloud and what are the discrepancies associates with ERP while running on the CC environment? This comparison is shown in Table 2 
International Journal on Cloud Computing: Services and Architecture (IJCCSA),Vol.3, No.3, June 2013

Table 2: Comparison of ERP systems before and after moving on to Cloud

\begin{tabular}{|c|c|c|}
\hline ERP Characteristics & $\begin{array}{c}\text { Before } \\
\text { Moving to } \\
\text { Clouds }\end{array}$ & $\begin{array}{c}\text { After } \\
\text { Moving to } \\
\text { Clouds }\end{array}$ \\
\hline Need for Technical IT Support for Fail over environments & $\checkmark$ & X \\
\hline Need for ERP Development Team & $\checkmark$ & X \\
\hline Need for extra hardware and software Resources and licenses & $\checkmark$ & X \\
\hline Need to configure latest technology updates & $\checkmark$ & X \\
\hline Need to arrange own extra power and cooling & $\checkmark$ & X \\
\hline Lack of computation and accuracy Trust & X & $\checkmark$ \\
\hline Lack of confidentiality & X & $\checkmark$ \\
\hline Lack of trust on security policies and access control rules & X & \\
\hline Daily Storage and Backup burden & $\checkmark$ & A \\
\hline Huge cost & $\checkmark$ & X \\
\hline High speed Internet connection & X & $\checkmark$ \\
\hline Subscription and registration charges & X & $V$ \\
\hline Need for requirement gathering and Elicitation & $\checkmark$ & $\bar{X}$ \\
\hline Need for Project Management & $\checkmark$ & X \\
\hline Need for Coding & $\checkmark$ & X \\
\hline Need for Testing & $\checkmark$ & X \\
\hline Need for Deployments & $\checkmark$ & X \\
\hline More Loss of control of any application or resources risks & X & $\checkmark$ \\
\hline $\begin{array}{l}\text { Conflicts between opposing goals of different clients, either } \\
\text { play it together if not need to separate them }\end{array}$ & X & \\
\hline Higher risks of Resource availability and failure & X & $\checkmark$ \\
\hline Lack of trust in data alteration before storing & X & $\checkmark$ \\
\hline Denial of Service attack in critical server health situations & X & 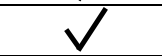 \\
\hline Higher risks of Stress, load and congestion & X & $\checkmark$ \\
\hline Difficult to audit & X & $V$ \\
\hline Monitoring of client logs and information by third party & X & 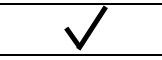 \\
\hline
\end{tabular}

As Table 2 shows, Cloud appears to be a promising future environment for ERP systems and it offers several benefits. According to the Table 2, hiring new development and technical support teams is a quite budget consuming process. On the other hand, the subscription charges of getting services from cloud are too low as compared to the charges of hiring new teams. Similarly, the cost of hardware arrangement, extra power, cooling, and accommodation can be significantly reduced after moving ERP onto Cloud. Furthermore, new technology updates and daily backup management burden are omitted after moving ERP onto cloud environment. According to our analysis, the share of cost (budget) reduction after moving ERP onto cloud is $50 \%$ and the reduction of daily backup burden is about $15 \%$. These shares cannot be neglected in any case and clearly invokes the justification of shifting ERP onto CC environment. The comparison of cost is further elaborated in Table 3. 
Table 3: Cost reduction when moving ERP onto Cloud

\begin{tabular}{|l|c|}
\hline Factors & \% Reductions \\
\hline Requirement Elicitation and gathering cost [29] & $25 \%$ \\
\hline Testing efforts $\{29,30]$ & $10 \%$ \\
\hline IT labor and development team cost [30] & $50 \%$ \\
\hline Capital Utilization Improvements [30] & $75 \%$ \\
\hline Technical IT Support Cost [30] & $40 \%$ \\
\hline Overall project cost [29] & $(30-40) \%$ \\
\hline
\end{tabular}

Table 2 also shows several challenges associate with the Cloud-based ERP such as lack of trust, loss of controls, security attacks, and auditing and monitoring control. However, the benefits of Cloud-based ERP are clearly superior as compared to these challenges. In the light of our comparison and analysis, we confidently suggest that migration of ERP onto the cloud environment is significantly beneficial for the companies, especially, for small and medium size companies that have some budget limitations. However, challenges depicted in Table 2 continue to be an extra ordinary future research for the Cloud community.

\section{A FRAMEWORK FOR CLOUD-BASED ERP SYSTEMS}

The second objective of this research is to propose a generic framework for ERP in CC environment in favor of SME. At the core of this framework is SaaS that makes ERP applications available on-demand either through a usage plan or timely interval payments. This feature attracts leading business organizations to adopt SaaS to avoid purchasing expensive software. The vendors may either make the software available on their own server or allow customers to download and use this particular app on the user device with the privilege to enable/disable the app when contract expires. The license includes single user usage or a group of user within an organization. Examples for SaaS ERP apps are: Human Resource Computerization, Invoice generation and billing, and Service management. Common applications such as accounting, CRM modules, MIS systems, HRM systems, and CMS use SaaS to deliver their models. Almost all SME will add SaaS as their major stratagem.

Two major issues in present ERP systems are "Industry functionality" and "Solution integration". The solution to these issues requires the Cloud to have different ERP instance for each industry. Each instance is supported with SOA technology stack for "Solution Integration" and is related to specific industry business need. Cloud computing infrastructure has eased-up our idea to implement robust and cost effective ERP solutions. By utilizing CC environment the ERP applications are presented in the form of services to be used accordingly. This approach has $90 \%$ saved the cost of software and hardware since ERP applications are presented in the form of SaaS and benefit from PaaS and IaaS characteristics in CC environment. In the light of the above discussion, we now propose a cloud-based framework that tackles the issues of industry functionality and solution integration in ERP systems. This framework is shown in figure 1 below. 


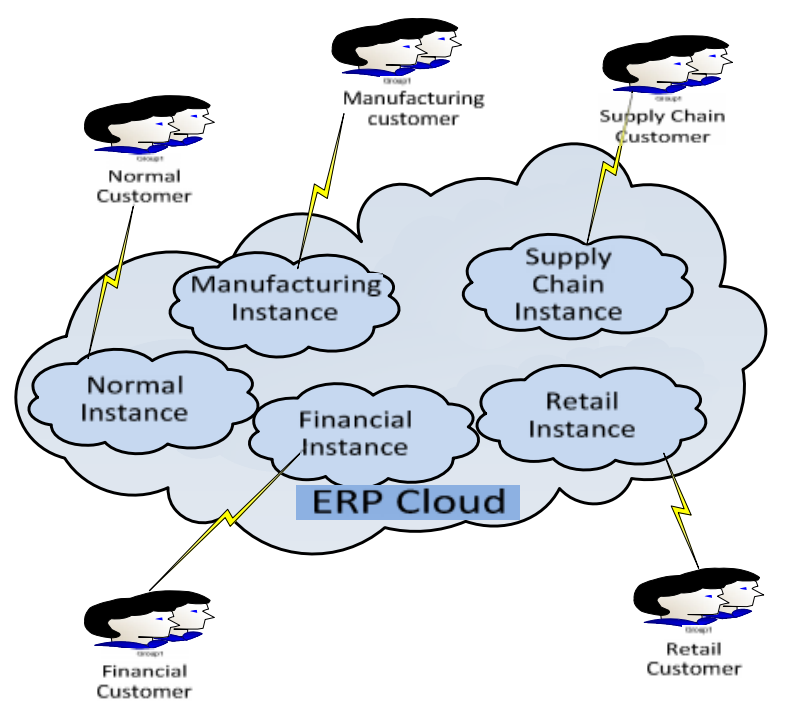

Figure 1: A Framework for Cloud-based ERP Systems

The solution to "Industry functionality" requires the Cloud to have different ERP instance for each industry. Customers can connect to the ERP instance that best fits their business need. This solution may require the ERP cloud provider to have not only instance per business category, but also per ERP tier, for example there should be Retail ERP mid-tier (e.g., Microsoft) and Retail ERP top-tier (e.g., Oracle). Solution integration can be managed through the utilization of two type of instances supported with SOA technology stack, SOA Application Tier and SOA DB Tier. The interaction between these two tiers is illustrated in figure 2. Most Enterprises will have this functionality; for example, in Oracle ERP, the ability to define multiple organizations and the relationships among them within a single installation of Oracle Applications is called multi organization or Multi-org. Multi-Org is the future technology to store the data of multiple organizations in a single Database instance.

SOA directly supports the enterprise-level processes and services that optimize investment and help build portfolios with enhanced capability. SOA provides the ability to easily orchestrate cross-functional business processes by improving the integration of ERP and non-ERP systems across a network. It speaks to the various requirements related to loosely coupled, standardsbased, and protocol independent distributed computing. Construction of SOA requires a highly distributable communications and integration backbone. This functionality is made available by the Enterprise Service Bus (ESB), which is an intelligent integration platform that employs Web services standards to support a broad variety of communications patterns over multiple transport protocols and deliver value-added capabilities for SOA applications. SOA requires that services are defined by a description language and have interfaces that perform useful business processes. It assumes that services can be dynamically located, invoked and re-combined. An SOA creates a collection of services that can communicate with each other using service interfaces to pass messages from one service to another, or coordinating an activity between services. 


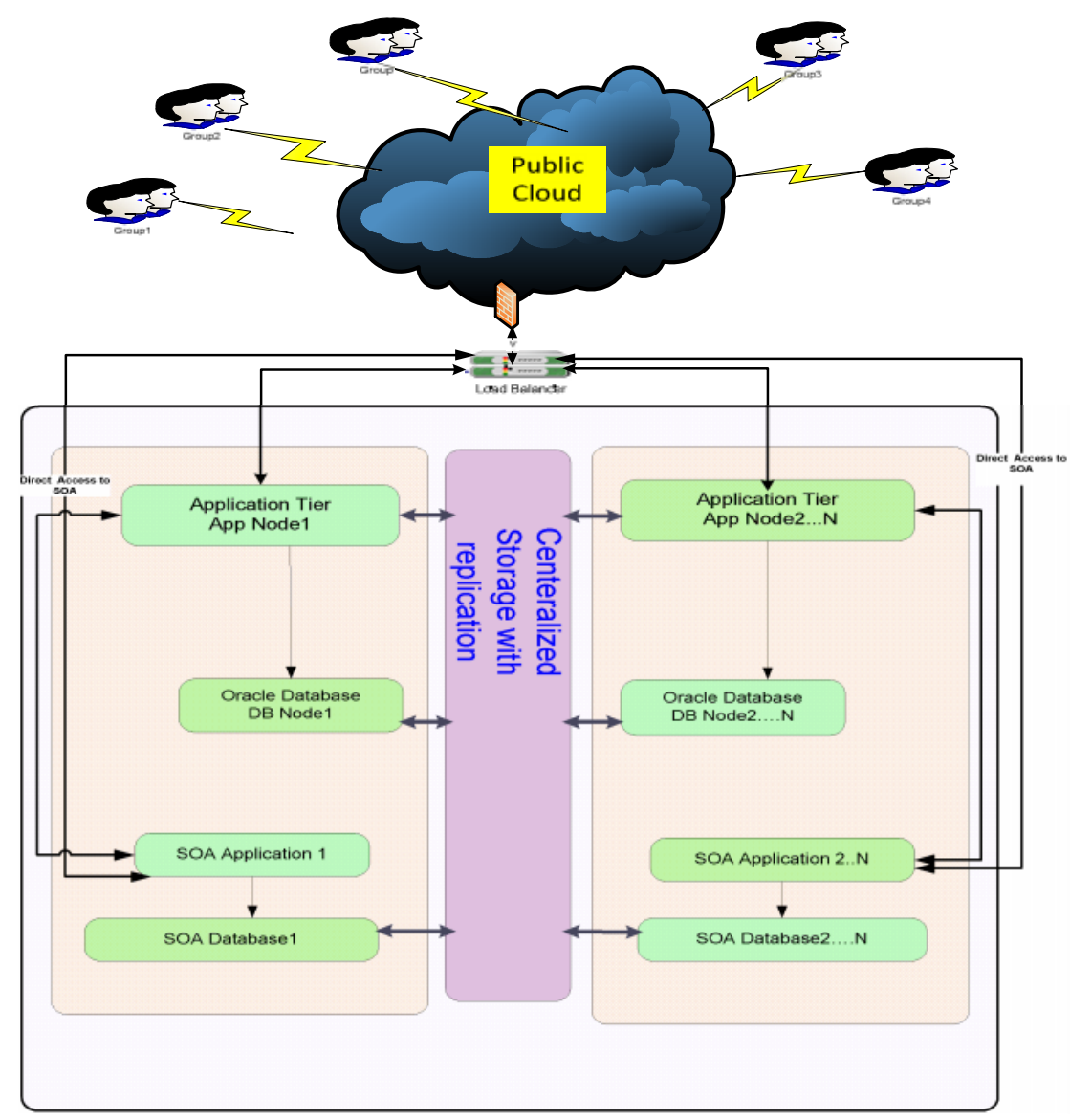

Figure 2: Working Interaction Between SOA Tiers

\section{CASE STUDY OF “AWAL” IT SERVICES COMPANY}

The third objective of this research is to conduct a case study to test the feasibility and efficiency of our proposed framework. For this purpose, we chose "Awal" IT Services Company to show that our proposed framework can help cope up current cost, industry functionality and solution integration issues in Awal's ERP system. Awal is not a newly established company, it is a part of Saudi Telecom Company (STC) that fills the gap between connectivity of infrastructure and IT services. It also facilitates end-to-end hosting services with user satisfaction. Awal provides a distinguished solution consulting due to its balanced focus on excellent people and knowledge expertise. It is fully capable of providing variety of customized services such as: Cloud Computing, Enterprise Security and Monitoring Solutions, Network Designs and Security Assessment, Operational Quality Assessment, IT Environment optimization, Business continuity solutions, Internet and Online Solutions.

Awal serves clients with dedicated hosting through a cloud having sufficient computational power. Awal's cloud creates data centers for smart cities, e-learning centers at educational institutions and hospital's diagnostic and record managements. Awal's cloud is enriched with latest IT instruments, services and technical support teams for troubleshooting. It provides different kinds of cloud services such as IaaS, CRM, Hosted Exchange Services, Call Center Services, educational and health services. Awal utilized IaaS for delivering several IT functions 
through subscriptions. Awal's IaaS possesses elasticity and availability with trusted IT partners. It implements CAPEX to OPEX based business models for providing best services to its clients. Awal provides a wide range of cloud services through its locally managed data centers with the following technology features and service benefits:

\section{Technology Features}

- Client's preferred Operating Systems

- Stable Virtual Machines

- Fully Redundant Architecture

- Web-Based Console

- Firewalls for security

- Compatibility support with already running applications

- Flexibility to cope new requirements.

- Technical service support (24/7) with guaranteed SLA

\section{Service Benefits}

- Enhanced cash flow: pay-per-use consumption ratio of desired model.

- Elasticity in new requirements and Flexibility in design

- Transparent services

- No limits for on demand resources

- Backup power control

Although Awal provides sufficient cost effective services, the deal is still too expensive for smaller industries. Awal's cloud services do not have any provision of customization and interaction among different kinds of industries. Some other challenges faced by Awal are: long transaction processing time, solution integration, and complexity of upgrading some functional modules on Cloud. Awal's services lack industry coordination and solution integration because they possess no satisfactory customization and integration among various organizations. Industry functionality and solution integration of Awal Company can be enhanced by utilizing our framework. The proposed framework is reasonably feasible for Awal Company as well as many other similar SME companies. Therefore, moving Awal's ERP system to the Cloud environment based on our proposed framework is an excellent deal to trigger future revenues. On the other hand, this framework is cost effective for growing industries.

\section{DISCUSSION}

Solution integration, industry functionality, security, availability of resources and execution capability are the prominent problems with cloud computing technology. The security issues represent about $75 \%$, the integration issues are about $61 \%$, and cloud's performance and resource availability issues are about 63\% as predicted by IDC Enterprise Panel in August 2008 (http://www.idc.com/). Furthermore, the issue of expensiveness and customization is around $50 \%$. The question of moving ERP onto CC environment is not affected negatively due to these issues because; security is the persistent issue whether ERP is on cloud environment or not. On the other hand, with Cloud-based ERP systems, performance will be enhanced due to the extraordinary computational power of cloud. Similarly, availability is directly proportion to number of resources, therefore, availability will be improved due to Cloud resource pooling.

Moving ERP onto CC environment is a good deal to save cost in the sense of there is no need for hiring new development and technical support teams and management of physical resources. This 
is because the subscription cost of cloud providers is many times lower as compared to the cost of self management of these resources by any medium or small company. Similarly, the cost of hardware arrangement, extra power, cooling, and accommodation can easily be reduced while moving ERP onto the Cloud. Furthermore, the hedge of daily backup and technology update can be escaped whiling moving the ERP onto CC environment. However, several challenges like lack of trust, loss of controls, multi-tendency, denial of services, auditing and monitoring controls are associates with Cloud-based ERP systems, but clearly the benefits are superior as compared to these types of challenges. Finally, we see that moving ERP onto CC environment is a significant and cost effective solution which is certainly beneficial for medium and smaller size organizations.

\section{CONCLUSIONS AND FUTURE WORK}

In this paper, we investigated the issue of how CC technology can be employed in ERP systems in favor of SME which have limited budget. We have conducted a comparative analysis for ERP before and after moving to CC environment and proposed a framework for Cloud-based ERP. Our investigation results confidently support moving ERP onto CC environment. Cloud-based ERP can reduce 50\% development team cost, $40 \%$ technical support team cost, $10 \%$ testing effort, $25 \%$ requirement elicitation, $15 \%$ burden of daily backup management, and $(30-40) \%$ cost of overall project expenditure. We also analyzed the case of "Awal" IT services company in order to shed the light on how ERP services are provided inside the company. It was clear that the way the company uses the Cloud to provide ERP services is too expensive for a small size organization. Our proposed framework is reasonably feasible for Awal Company and can enhance the industry functionality and solution integration in this company as well as similar SME companies.

The presented framework is cost effective for growing industries and it is sufficiently to trigger future revenues. Due to its economical cost, we believe that the proposed framework will be the ultimate choice of small and medium sized organizations in near future. However, Cloud security concerns such as data encryption prior to store on the Cloud, secure network linkage, secure allocation of resources under trusted users as well as trusted processes, and safe movement of transactions among different cloud nodes are still challenges in front of future research. Since ERP applications are web based, ERP present and future systems necessarily require standardized set of security policies.

\section{REFERENCES}

[1] Sayed Hashimi. (2003). Service-Oriented Architecture Explained. O’Reilly ONDotnet.com.

[2] T. Peeyush. ( 2008). Service Oriented Approach for ERP Integration. Oracle Corporation.

[3] Peter Mell and Timothy Grance. (2011). The NIST Definition of Cloud Computing Standard and Technology, U.S department of Commerce.

[4] D. Krafzig, K. Banke, and D. Slama. (2004). Enterprise SOA: Service-Oriented Architecture Best Practices. Prentice Hall, 1st Edition.

[5] Richard Veryard. (2008). A Simple History of SOA. Independent Guidance for Service Architecture and Engineering, Everware-CBDI.

[6] Wei-Tek Tsai, Xin Sun and Janaka Balasooriya. (2010). Service-Oriented Cloud Computing Architecture. Seventh International Conference on Information Technology: New Generation, pp. 684-689. 
International Journal on Cloud Computing: Services and Architecture (IJCCSA),Vol.3, No.3, June 2013

[7] Roopali Goel and Vinay Rishiwal. (2012). Cloud Computing and Service Oriented Architecture. International Journal of Recent Technology and Engineering (IJRTE), Vol. 1, Issue 1, pp. 137-139.

[8] D. Galani, E. Gravas and A. Stavropoulos. (2010).The Impact of ERP Systems on Accounting Process. World Academy of Science, Engineering and Technology, Vol. 66, pp. 418-423.

[9] C. Holsapple and M. Sena. (1999). Enterprise System for Organization Decision Support: A research Agenda. Proceedings of AMCIS.

[10] Ahmed A. Elragal and Ayman M. Al-Serafi. (2011). The Effect of ERP System Implementation on Business Performance: An Exploratory Case-Study. Communications of the IBIMA, Vol. 2011, Article ID. 670212.

[11] P.T. Kale, S.S. Banwait, and S.C. Laroiya. (2008). Enterprise Resource Planning Implementation in Indian SMEs $\square$ : Issues and Challenges. 12th Annual International Conference of Society of Operation Management, pp. 242-248.

[12] S.G. Yaseen. (2009). Critical Factors Affecting Enterprise Resource Planning Implementation: An Explanatory Case Study. International Journal of Computer Science and Network Security IJCSNS, Vol. 9, No. 4, pp. 359-363.

[13] Toni M. Somers and Klara Nelson. (2001). The Impact of Critical Success Factors across the Stages of Enterprise Resource Planning Implementations. Proceedings of the 34th Hawaii International Conference on System Sciences, pp. 8016, Vol. 8.

[14] X. Yao and M.B. Dan-Rognlie. (2007). Distributed Transaction Management in SOA-based System Integration. Mater Thesis, IT University of Copenhagen.

[15] Huaglory Tianfield. (2011). Cloud Computing Architecture. 2011 IEEE International Conference on Systems, Man, and Cybernetics (SMC), pp. 1394-1399.

[16] D.D. Giacomo and T. Brunzel. (2010). Cloud Computing Evaluation- How it Differs to Traditional IT Outsourcing", Master Thesis, Jonkoping University.

[17] Sean Marston, Zhi Li, Subhajyoti Bandyopadhyay, Juheng Zhang and Anand Ghalsasi. (2011). Cloud Computing — The Business Perspective", Journal Decision Support Systems, Vol. 51, Issue. 1, pp. 176189.

[18] G. Suciu, O. Fratu, S. Halunga, C.G.Cernat, V.Poenaru and V.Suciu. (2012). Cloud Consulting: ERP and Communication Application Integration in Open Source Cloud Systems. $19^{\text {th }}$ Telecommunication Forum (TELFOR), Serbia, Belgrade, pp. 578-581.

[19] M. Baktashmotlagh, A. Bigdeli and B.C.Lovell. (2011). Dynamic Resource Aware Sensor Networks: Integration of Sensor Cloud and ERPs. 8th IEEE International Conference on Advanced Video and Signal Based Surveillance, pp. 455-460.

[20] W.H. Tsai, S.P. Chen, E.T.Y. Hwang and J.L. Hsu. (2010). A Study of the Impact of Business Process on the ERP System Effectiveness. International Journal of Business and Management, Vol. 5, No. 9.

[21] J. Isse. (2010). Cloud Computing and the Innovation Process of Technology Consulting Services: the Case of Accenture. Master Thesis, Blekinge Institute of Technology.

[22] R. Saleem. (2011). Cloud Computing Effect On Enterprises in term of cost and security, Mater Thesis, Lund University.

[23] Shehab M.W, Sharp L, et. al. (2004). Enterprise resource planning, Business Process Management Journal, Vol. 10 No. 4, 2004, pp. 359-386, Emerald Group Publishing Limited 1463-7154. DOI $10.1108 / 14637150410548056$.

[24] Mabert, V.A., Soni, A. and Venkataramanan, M.A. (2001). Enterprise resource planning: common myths versus evolving reality. Business Horizons, pp. 69-76.

[25] Coffey, M., Kelly, L. and Parks, M. (2000). Enterprise Resource Planning (ERP). http://personalpages.geneseo.edu mpp2/erppaper.htm (accessed 30 October 2001). 
International Journal on Cloud Computing: Services and Architecture (IJCCSA) ,Vol.3, No.3, June 2013

[26] Themistocleous, M., Irani, Z. and O'Keefe, R. (2001). ERP and application integration: exploratory survey. Business Process Management Journal, Vol. 7 No. 3, pp. 195-204.

[27] Thomas Erl. (2007). SOA Principles of Service Design. Prentice Hall.

[28] Youssef, A. (2012). Exploring Cloud Computing Services and Applications. Journal of Emerging Trends in Computing and Information Sciences (JEMCIS). 3(6):838-847.

[29] Peter C. (2009). Clouding Computing: More Than a Virtual Stack. Platform Research, salesforce.com, Torrance, California, USA

[30] IIIT, Hyderabad (2010). Cloud Computing for E-Governance, A white Paper. 